\title{
"Fou rire prodromique" as the presentation of pontine ischaemia secondary to vertebrobasilar stenosis
}

F de A A Gondim, B J Parks, S Cruz-Flores

\begin{abstract}
"Fou rire prodromique" (prodrome of crazy laughter) is a rare form of pathological laughter of uncertain pathophysiology. A patient is presented with pathological laughter as the first manifestation of pontine ischaemia due to vertebrobasilar stenosis. A 65 year old man developed uncontrollable and unemotional laughter for almost an hour followed by transient right facial-brachial paresis. He had fluctuation of laughter, right facial brachial paresis, and occasional crying. Magnetic resonance imaging, magnetic resonance angiogram (MRA), and an angiogram showed small left pontine and cerebellar infarcts, left vertebral artery occlusion, and right vertebral and basilar artery stenosis. His condition deteriorated to bilateral brain stem infarction and he died. Necropsy confirmed the extensive brain stem infarction. Pathological laughter can be the very first presenting manifestation of ischaemia of the ventrotegmental junction of the upper pons. It is hypothesised that the pathological laughter in this patient was secondary to ischaemic ephaptic stimulation of the descending corticopontine/ bulbar pathways.

(F Neurol Neurosurg Psychiatry 2001;71:802-804)
\end{abstract}

Keywords: pathological laughter; fou rire; brain stem stroke; brain stem ischaemia

Pathological laughter is associated with a wide range of neuropsychiatric disorders. "Fou rire prodromique" is one of its forms and was first described by Féré in $1903 .{ }^{1}$ It can be literally translated as "prodrome of crazy laughter" and is defined as pathological laughter preceding a major apopletic event. ${ }^{2}$ It has been reported rarely and its mechanisms are still not fully understood.

This type of pathological laughter has been reported as the presentation of bilateral thalamic hemorrhages, ${ }^{3}$ strokes in the right lenticular nucleus/internal capsule, ${ }^{4}$ and left capsule/lenticular nucleus/ insula due to left internal carotid occlusion, ${ }^{56}$ left capsule/ thalamus, ${ }^{7}$ or left putamen/internal capsule. ${ }^{2}$ Other reports described pathological laughter after hemiparesis in pontine strokes or heralding left temporal ischaemia..$^{8-12}$

We present the first description of pathological laughter as the actual initial symptom of pontine stroke secondary to severe vertebrobasilar artery stenosis. This report has been published in abstract form. ${ }^{13}$

\section{Case report}

A 65 year old right handed man suddenly developed uncontrollable, persistent, unemotional laughter which lasted for almost an hour. He felt exhausted because of the laughter and then developed right hand and facial paresis that resolved almost completely on arrival at the emergency room.

He had hypertension for 30 years and was taking $60 \mathrm{mg}$ nadolol every day. He denied smoking and used to drink a few beers. He was in good health, except for occasional nonpositional dizziness for 2 weeks and one spell of vertigo with an increaase in his blood pressure before his admission. He denied dysphagia, diplopia, amaurosis, headache, dysphonia, dysarthria, hearing loss, loss of consciousness, and head or neck trauma.

He was admitted to a local hospital where a head CT was reported "negative". He continued to have brief spells of laughter alternating with crying but with no paresis. Next day, a neurologist documented normal examination and a preliminary diagnosis of left hemispheric transient ischaemic attacks versus gelastic epilepsy or anxiety was made. A few hours later, he developed right brachial-facial paresis. Brain MRI/MRA showed small infarcts in the midlateral pons (ventrotegmental junction at the level of the pontomesencephalic junction) and left cerebellar hemisphere (fig 1). Occlusion of the left vertebral artery and narrowing of the right vertebral and basilar arteries was also seen. Intravenous heparin was started.

His condition deteriorated and cerebral angiography was performed. Neurological examination disclosed dysarthria, bilateral, reactive, $2 \mathrm{~mm}$ pupils, desconjugated gaze, gaze evoked upbeat nystagmus, decreased corneal reflex on the left, preserved gag reflex, right hemiparesis ( $2 / 5$ in the upper limb, $4 / 5$ in the lower limb), decreased tone, brisk reflexes on the right, right Babinski's sign, and decreased pinprick and touch on the right. 


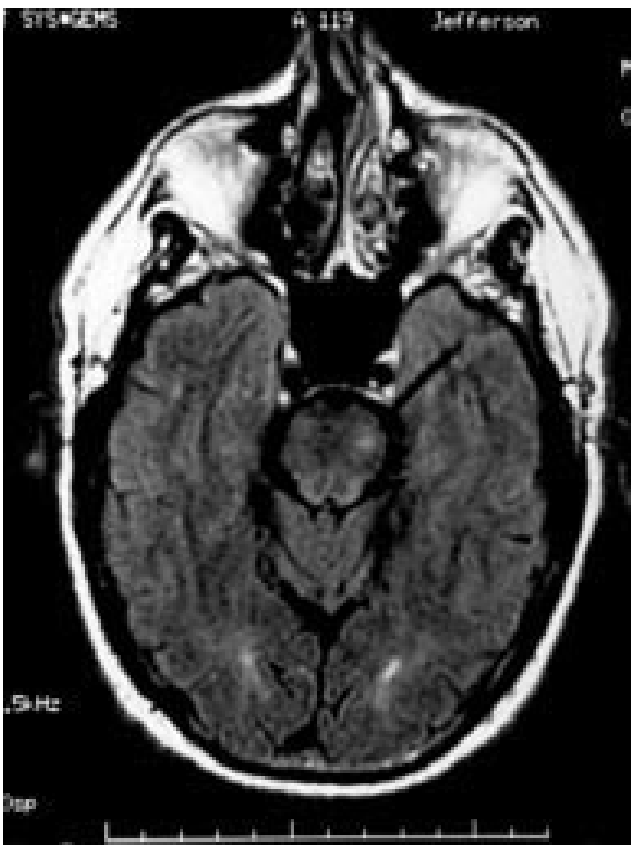

Figure 1 Brain MRI (FLAIR) of the pontomesencephalic junction shows ischaemia of the left pons in the ventrotegmental junction.

The cerebral angiogram showed occlusion of the left vertebral artery, severe right vertebral stenosis just distal to the posterior inferior cerebellar artery, and minimal retrograde flow from the posterior communicating to the basilar artery. Intravenous heparin was continued. He subsequently developed a decreased level of alertness, left internuclear ophtalmoplegia, right gaze deviation, and severe dysphonia. Occasional crying but no laughter was seen until the next day, when he progressed to a locked in state and coma. A vertebrobasilar angioplasty was attempted but was unsuccessful. Head CT showed diffuse brain stem swelling with hydrocephalus. He continued to deteriorate and died.

\section{Postmortem results}

His brain weighed $1410 \mathrm{~g}$.

CENTRAL NERVOUS SYSTEM

There had been an acute infarct in the midbrain (left greater than right), pons (left greater than right), left medulla, and cerebellum (diffuse on the left and multifocal on the right) with moderate hydrocephalus. The left vertebral artery was occluded greater than $95 \%$ by atherosclerosis and thrombus, the right vertebral artery was narrowed by $60 \%$ by atherosclerotic plaque (fig 2).

\section{HEART}

Coronary artery disease was present (right coronary $50 \%$ occluded, left anterior descending $30 \%$ occluded and left circumflex $60 \%$ occluded). No intracardiac thrombus was present. There were atherosclerotic changes in the aorta.

\section{Discussion}

We present a case of pathological laughter marking the onset of pontine ischaemia that progressed to extensive brain stem infarction.

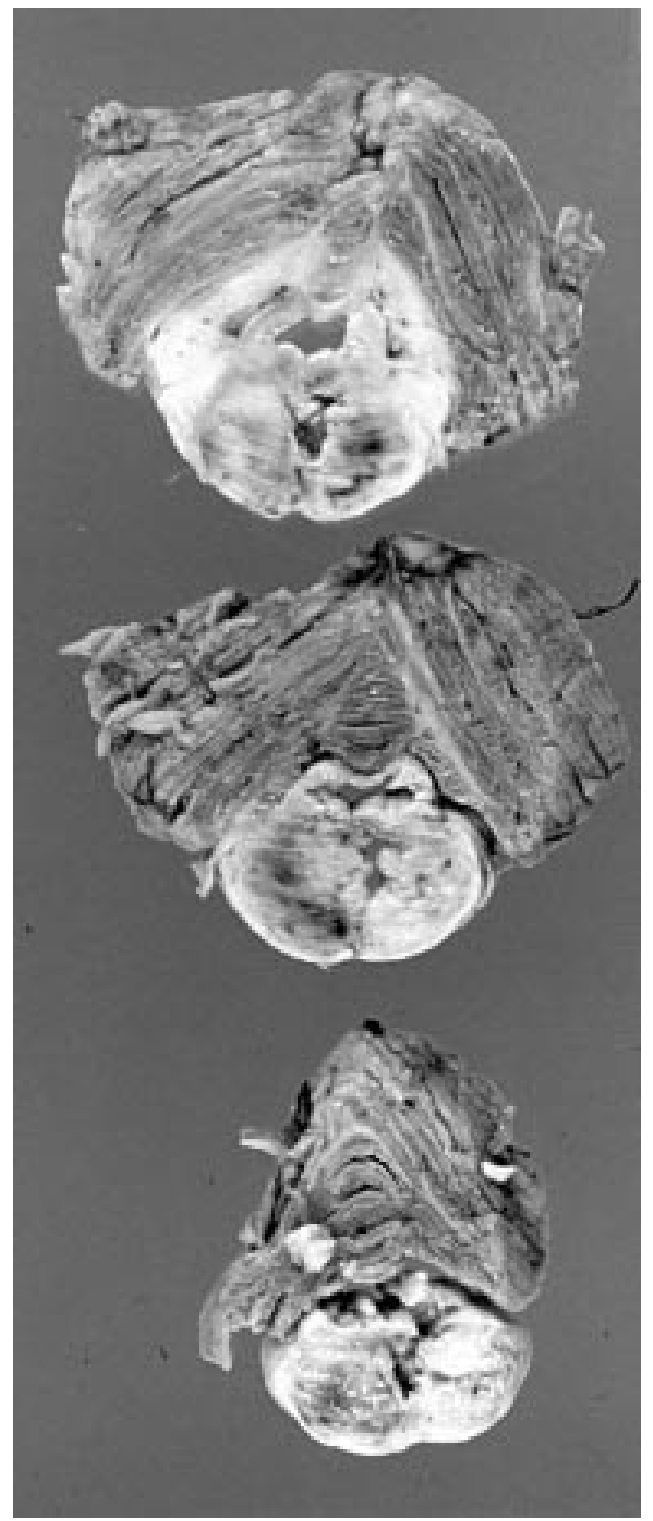

Figure 2 Pathology specimens from the lower pons up to the pontomesencephalic junction show the presence of bilateral infarction of the pons, left more pronounced than the right.

This is the first description of "Fou rire prodromique" as the actual first presenting symptom of pontine ischaemia, as in the previous reports laughter followed the onset of focal deficits. ${ }^{9-12}$ In the original paper by Féré, pathological laughter followed other deficits such as hemiplegia, and although suggested by its French name, "Fou rire prodromique", the symptom was not a prodrome. ${ }^{1}$

Pathological laughter has been reported after strokes at different locations: left or bilateral pons, striatocapsular infarcts, left temporalparietal lobe, thalamus, caudate, or lenticular nuclei. ${ }^{4-12}$ This symptom has been linked to catastrophic conditions such as carotid artery dissection, ${ }^{6}$ cerebellar haemorrhages, ${ }^{14}$ and brain tumours, ${ }^{15}$ and also to innocuous interventions such as intravenous infusion of valproate. ${ }^{16}$

The mechanisms leading to pathological laughter are not understood. We hypothesised 
that our patient initially had ischaemia of the ventrotegmental junction of the pons because pathological laughter was followed by motor involvement of the arm and face. ${ }^{17}$ This was confirmed by the MRI findings, which showed a left pontine infarct. With the extension of the ischaemia, crying dominated the picture and sensory deficits, eye movement disturbance, and later, a locked in state became apparent.

Pathological laughter can be indistinguishable from gelastic seizure after temporal lobe infarction. ${ }^{12}$ In subcortical ischaemia, pathological laughter may be the result of a "disconnection syndrome", due to release of pathways previously under tonic inhibition. ${ }^{2}$ Poeck proposed that there is tonic and phasic control of the facial muscles and that pathological laughter is the result of the loss of tonic control. ${ }^{2}$

In our patient we think that pathological laughter was secondary to transverse (axon to axon) ephaptic transmission within a partially demyelinated focus in the pontomesencephalic ventrotegmental junction caused by ischaemia. A similar mechanism has been proposed as the aetiology of involuntary tonic limb spasms after brain stem infarctions. ${ }^{18}$

Bilateral subcortical strokes lead to pathological laughter and a pseudobulbar state with emotional incontinence. However, pathological laughter after a unilateral lesion or as a result of ongoing ischaemia, is most likely the result of a positive phenomenon - that is, ephaptic transmission, with stimulation of pathways leading to bilateral activation of the facial muscles. ${ }^{4-12}$ There are distinct pathways for the control of voluntary and emotional facial movements. Because emotional laughter is not abolished in the locked in state, ${ }^{19}$ we speculate that pathological laughter in our patient was due to involvement of the terminal portion of the emotional pathway. This pathway seems to synapse in the pontine tegmentum, where interneurons for the facial nerve are located. ${ }^{20}$ Although it could be argued that more extensive ischaemia was the cause of the symptom, involving the brain stem, or temporal lobe, or both, our speculation is supported by the initial limited imaging findings, and the neurological findings early in the course of the disease of our patient.

Laughter is a rather complex series of facial movements and involves coordination of transient breathing arrest in addition to activation of the facial nuclei. Data in monkeys showed that emotional pathways probably originated in the cingulate gyrus and its axons run intermingled with axons responsible for the movements of upper limbs and control of respiratory muscles. ${ }^{21}$ The centres for these complex activities are closely related to centres that are known modulators of breathing, cardiovascular, and autonomic functions. Areas in the lateral tegmentum that could be involved in these functions include the parabrachial nuclei,
Kolliker-Fuse nucleus (ventral to parabrachial nuclei), and raphe nuclei. The raphe nuclei are particularly rich in serotonin and are the source of the serotoninergic fibre system. Pathological emotionalism after strokes seems to involve serotoninergic pathways, which could explain the late pathological crying in our patient. ${ }^{22}$

In summary, we presented a case of pathological laughter heralding extensive brain stem ischaemia in whom the laughter was probably secondary to ephaptic transmission in the descending corticopontine/bulbar pathways within the ischaemic region. Although rare, this symptom can be the early sign of catastrophic vertebrobasilar ischaemia and should be considered in the differential diagnosis as it is a potentially treatable condition. ${ }^{11}$ This work was presented at the 125th Annual Meeting of the
American Neurological Association, 15-18 October 2000, Boston, MA, USA.

1 Féré MC. Le fou rire prodromique. Rev Neurol (Paris) 1903;7:353-8

2 Poeck K. Pathologic laughter and crying. In: Vinken PJ, Bruyn GW, Klawans HL, eds. Handbook of clinical neurology. Amsterdam: Elsevier, 1985:219-26.

3 Andersen. Crise de rire spasmodique avant déc'es: Hémorragie thalamique double. Fournal Belge de Neurologie et de Psychiatrie 1936;36:223-7.

4 Burzio F. Emiplegia con dolori ed accessi di riso spasmodico: da rammollimento del nucleo lenticolare. Analidi Freniatria e Scienze Affini Freniat Sciz Affini 1900;10:140-8

5 Carel C, Albucher JF, Manelfe C, et al. Fou rire prodromique heralding a left internal carotid occlusion. Prroke $1997 \cdot 28 \cdot 2081-3$

6 Osseby G, Manceau E, Huet F. "Fou rire prodromique" as the heralding symptom of lenticular infarction, caused by dissection of the internal carotid artery in a 12-year-old boy. European fournal of Paedratic Neurology 1999;3:133-6.

7 Ceccaldi M, Milandre L. A transient fit of laughter as the inaugural symptom of capsular-thalamic infarction. Neurology 1994;44:1762.

8 Wali GM. "Fou rire prodromique" heralding a brainstem stroke. $\mathcal{F}$ Neurol Neurosurg Psychiatry 1993;56:209-10.

9 Tei H. Pontine infarction due to basilar artery stenosis presenting as pathological laughter. Neuroradiol 1997;39: $190-1$.

10 Mouton O, Rémy A, Cambon H. Rire spasmodique par lésion unilatérale du tronc cérébral. Rev Neurol (Paris) 1994;150:302-3.

11 Couderq C, Drouineau J, Rosier MP. Fou rire prodromique d'une occlusion du tronc basilaire. Rev Neurol (Paris) 2000;156:281-84.

12 Lago A, Beltran I, Ferrer JM. Episodio transitorio de risa como sintoma inicial de ictus isquémico. Rev Neurol 1997; 25:239-41.

13 Gondim F, Parks BJ, Cruz-Flores S. Fou rire prodromique: an unusual presentation of severe vertebrobasilar stenosis. Ann Neurol 2000;48:423.

4 Doorenbos DI, Haerer AF, Payment M, et al. Stimulusspecific pathological laughter: a case report with discrete unilateral localization. Neurology 1993;43:229-30.

15 Ceccaldi M, Donnet A, Grisoli F, et al. Fou rire prodromique et tumeur prérolandique. Rev Neurol (Paris) 1995;151:206-8.

16 Jacob PC, RP Chand. Pathological laughter following intravenous sodium valproate. Can f Neurol Sci 1998;25:252-3.

17 Bassetti C, Bogousslavsky, Barth A, et al. Isolated infarcts of the pons. Neurology 1996;46:165-75.

18 Wilson SAK. Some problems in neurology: II. Pathological laughter. F Neurol Psychopathol 1924;16:299-33.

19 Feldman MH. Physiological observations in a chronic case of locked-in syndrome. Neurology 1968;18:1166-79.

20 Topper R, Kosinski C, Mull M. Volitional type of facial palsy associated with pontine ischaemia. F Neurol Neurosurg Psychiatry 1995;58:732-4.

21 Morecraft RJ, Louie JL, Herrick JL, et al. Cortical innervation of the facial nucleus in the non-human primate. Brain 2001;124:176-208.

22 Andersen $\mathrm{G}$, Ingeman-Nielsen $\mathrm{M}$, Vestergaard $\mathrm{K}$, et al. Pathoanatomic correlation between poststroke pathological crying and damage to brain areas involved in serotonergic neurotransmission. Stroke 1994;25:1050-2. 